\title{
Voltage-current characteristics of high-current glow discharges
}

\author{
K. Takaki, ${ }^{\text {a) }}$ D. Taguchi, and T. Fujiwara \\ Department of Electrical and Electronic Engineering, Iwate University, Ueda 4-3-5, \\ Morioka 020-8551, Japan
}

(Received 9 October 2000; accepted for publication 5 March 2001)

\begin{abstract}
The voltage-current characteristics of glow discharges in gas mixture $\left(\mathrm{N}_{2}: \mathrm{O}_{2}=8: 2\right)$ at a pressure of 10 Torr were obtained with the discharge current up to $150 \mathrm{~A}$. Parallel-plane electrodes with a diameter of $10.7 \mathrm{~cm}$ and a discharge chamber with co-axial geometry were used to produce glow discharge with high current. The glow discharge voltage was almost constant until the whole surface of the cathode was covered with glow, i.e., until the discharge current became $3.7 \mathrm{~A}$ in our experimental condition (a normal glow discharge mode). The voltage, however, increased with the current when the glow covered over the cathode (an abnormal glow discharge mode). The electron density in positive column of the high-current glow discharge was obtained to be $3 \times 10^{11} \mathrm{~cm}^{-3}$ from Langmuir probe measurements. (C) 2001 American Institute of Physics.
\end{abstract}

[DOI: $10.1063 / 1.1369612]$

Glow discharge plasmas have been indispensable for fabricating integrated circuits, materials, and surface structures, and modifying surface properties. This is because of the unmatched capability of glow discharge plasma devices, in providing a vast array of chemically active spices, selective ion flux, low gas temperature, and uniform reaction rate over a relatively large area. Alot of processes in a direct current (dc) glow plasma are governed by the voltagecurrent $(V-I)$ characteristics of the discharge. ' For example, the value of $E / N$, the ratio of electric field to neutral gas density, determines the rate constant of chemical reactions.

The glow plasma has been mainly produced in low pressure, for example, capacitively coupled plasma, inductively coupled plasma, electron cyclotron resonance, and dc glow plasma. Recently, the glow plasmas have been produced in higher gas pressure (from a few Torr to atmospheric pressure) for the purpose of other industrial applications, to simplify the whole system by reducing a matching unit and/or a vacuum pump..$^{2-6}$ Some of these plasmas are produced by $\mathrm{dc}$ or dc-like glow discharge. ${ }^{2,6}$ Our objective is production of a high density, large volume and low gas temperature plasma utilizing a pulse glow discharge with high-current in gas pressure from a few Torr to a hundred Torr. This letter describes experimental studies on the $V-I$ characteristics of a transient or pulsed glow discharge with high current up to 150 A after static breakdown of gas mixture $\left(\mathrm{N}_{2}: \mathrm{O}_{2}\right.$ $=8: 2$ ).

The electrode plates with rounded edges were made of brass and set in the discharge chamber. ${ }^{2}$ The overall diameter and thickness of the electrodes are $10.7 \mathrm{~cm}$ and $1.5 \mathrm{~cm}$, respectively. The one electrode is connected to a $1.89 \mu \mathrm{F}$ capacitor which is charged negatively up to high voltage. The other electrode is grounded through the damping resistors. The gap spacing between the electrodes was kept at 1 $\mathrm{cm}$. The pressure of gas mixture $\left(\mathrm{N}_{2}: \mathrm{O}_{2}=8: 2\right)$ was 10 Torr in the discharge chamber. The gas in the chamber was exchanged after every discharge and the electrodes were not

\footnotetext{
a)Electronic mail: koichit@mcmaster.ca
}

cooled in the experiment. The $1.89 \mu \mathrm{F}$ capacitor was slowly charged with nearly constant current of $0.5 \mathrm{~mA}$. Carborundum damping resistors were used to control the discharge current. The return current flows into the ground through 12 stainless rods. The breakdown voltage was accurately measured using a resistive voltage divider with 1000:1 ratio and $4 \mathrm{~ms}$ time constant. The value of time constant is sufficiently small because the time for charging the capacitor is of the order of a second. The discharge current was measured by means of a Pearson Model 110A current transformer and a Sony-Tektronix Model 540 digital oscilloscope. Then, the signal is transmitted to a personal computer through a GP-IB cable in order to calculate the gap voltage $v(t)$ using the following circuit equation:

$$
v(t)=V_{\mathrm{bd}}-\frac{1}{C} \int i(t) d t-R \times i(t)-L \frac{d i(t)}{d t},
$$

where $V_{\mathrm{bd}}$ and $i$ are breakdown voltage and current, respectively; $C, R$, and $L$ are the capacitance, resistance, and inductance of the circuit, respectively. ${ }^{7,8}$ The inductance $L$ was determined from a period of the $L-C$ oscillation in the prior experiment.

The damping resistor was altered from 1 to $200 \Omega$ in order to obtain the $V-I$ characteristics in wide current range. In order to obtain the electron density and temperature using Langmuir probe, we repeated the measurement of the probe current wave forms in pulsed glow plasma at various probe bias voltages, and probe current-bias voltage curves were drawn using them at various times. The probe tip is made of tungsten with $0.2 \mathrm{~mm}$ diameter and $3 \mathrm{~mm}$ length, ${ }^{9}$ and is placed at the middle between the electrodes. The probe was positioned at the middle between the electrodes.

Representative voltage and current wave forms of highcurrent transient glow discharge at damping resistor of $1 \Omega$ are shown in Fig. 1. After a static breakdown, marked (1) on the trace, the discharge current increases up to $150 \mathrm{~A}$, and the gap voltage decreases from the breakdown voltage to the value of a quasi-stable step, marked (2), as the discharge develops. The time duration from (2) to (3) indicates that the 


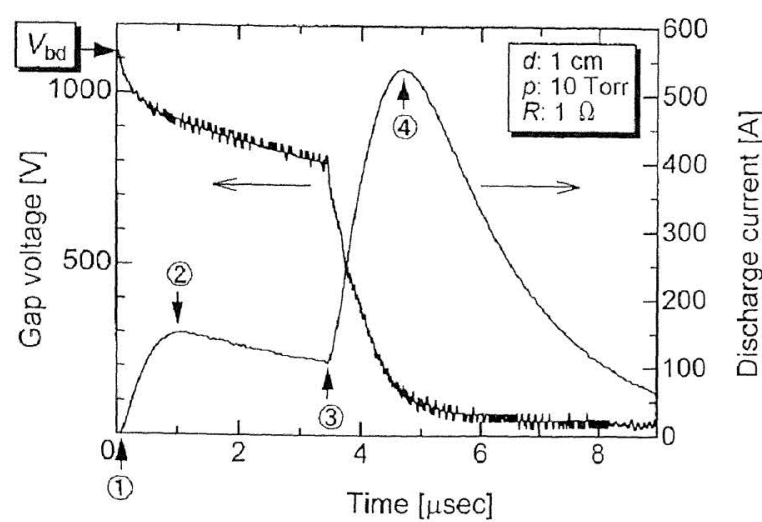

FIG. 1. Voltage and current wave forms of high-current transient glow discharge. $P=10$ Torr, $d=1 \mathrm{~cm}$, and $R=1 \Omega$.

discharge mode is the transient glow. In this case, the glow voltage decreases from 920 to $800 \mathrm{~V}$ with decreasing glow current from 150 to $107 \mathrm{~A}$. At the time marked (3), an appearance of a luminous spot on the cathode surface was observed by the image-converter camera photographs. ${ }^{8}$ After then, the voltage collapses to a few tens of volts with glowto-arc transition in time from (3) to (4). After the time marked (4), the arc discharge current decreases at a time constant of $R C$.

Figure 2 shows the $V-I$ characteristics obtained from Fig. 1. The $V-I$ characteristics have two positive slopes; the first is the time from (2) to (3) and the second is the time after (4). These correspond the characteristics of the glow discharge and the arc discharge, respectively. The two negative slopes mean the transient phases; Townsent-to-glow transition (1)-(2) and glow-to-arc transitions (3)-(4)).

Figure 3 shows the $V-I$ curves of the glow mode at various values of the resistance $R$ of the damping resistor. The broken line shows the interpolation by an exponential function. In the case of $R=1,3,9 \Omega$, the glow mode is transient, which means glow-to-arc transition occurs after the glow mode. In this case, the duration of glow modes are 3.5, 3.0 , and $20 \mu \mathrm{s}$, respectively. When $R>18 \Omega$, the glow-to-arc transitions do not occur, therefore the glow mode durations are almost determined by the time constant $C R(=1.89 R$ $[\mu s])$ of the apparatus. The empirical formula of current density $j_{\mathrm{ng}}: 10$

$$
j_{\mathrm{ng}}=240 \times 10^{-6} \mathrm{p}^{2}\left(\mathrm{~A} / \mathrm{cm}^{2}\right)
$$

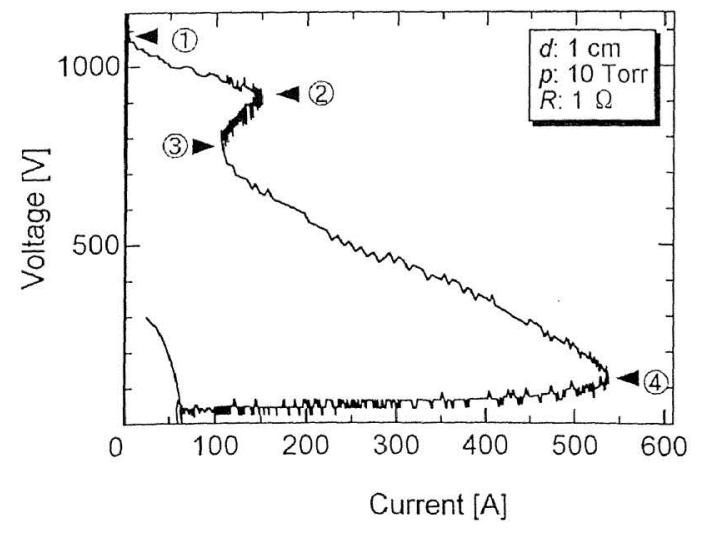

FIG. 2. Voltage and current characteristics $(V-I)$ of high-current transient glow discharge. $P=10$ Torr, $d=1 \mathrm{~cm}$, and $R=1 \Omega$.

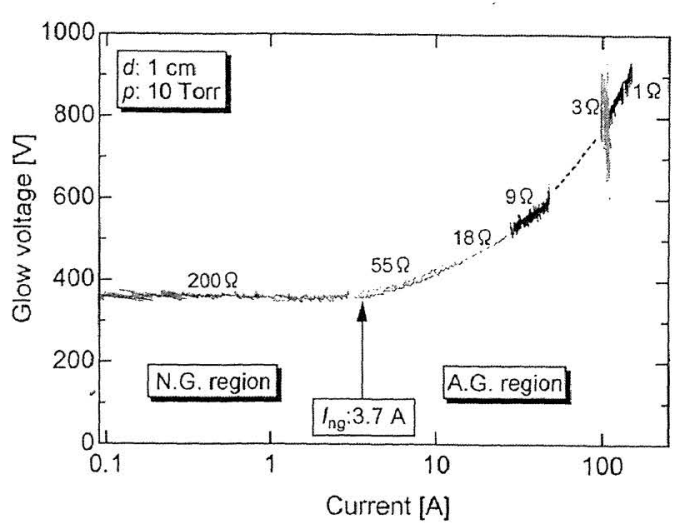

FIG. 3. $V-I$ characteristics of glow mode. $P=10$ Torr and $d=1 \mathrm{~cm}$.

for $\mathrm{Cu}$-air low pressure normal glow, where $p$ is gas pressure. The total surface area of the electrode $10.7 \mathrm{~cm}$ in diameter is obtained to be $155 \mathrm{~cm}^{2}\left(=76 \mathrm{~cm}^{2}\right.$ plane area +79 $\mathrm{cm}^{2}$ rounded edge). Therefore, when the whole cathode surface is surrounded by normal glow, the value of the current is obtained to be $3.7 \mathrm{~A}$, which is indicated as $I_{\mathrm{ng}}$ by an arrow in Fig. 3. The glow voltage is almost constant in the case of current lower than $3.7 \mathrm{~A}$ while it increases with increasing current in a range higher than $3.7 \mathrm{~A}$. This fact implies that the discharge changes from a normal glow to an abnormal glow at the discharge current of $3.7 \mathrm{~A}$.

The value of $E / p$, on the plateau of the $V-I$ curve, in the positive column of the glow discharge was deduced from the measured values of the glow voltage. We obtained $285 \mathrm{~V}$ as the cathode fall, which was determined by "zero length voltage" extrapolating the potential distribution across glow discharge to zero electrode separation., ${ }^{7, I}$ In the normal glow mode $(I<3.7 \mathrm{~A})$, the value of $E / p$ is obtained to be 8.6 $\mathrm{V} / \mathrm{cm} /$ Torr and independent of the discharge current. At discharge current of $144 \mathrm{~A}$ (in abnormal glow mode), the $E / p$ is $61.6 \mathrm{~V} / \mathrm{cm} /$ Torr. The electron density of the positive column $N_{e}$ can be estimated using the following formula:

$$
N_{e}=\frac{j}{e W},
$$

where $j, e$, and $W$ are current density, electron charge, and electron drift velocity, respectively. The parameter $W$ is calculated as a function of $E / N,,^{12}$ and it is $4.5 \times 10^{6} \mathrm{~cm} / \mathrm{s}$ for normal glow mode and $20.5 \times 10^{6} \mathrm{~cm} / \mathrm{s}$ for abnormal glow at current of $144 \mathrm{~A}$. The current density $j$ in the abnormal glow

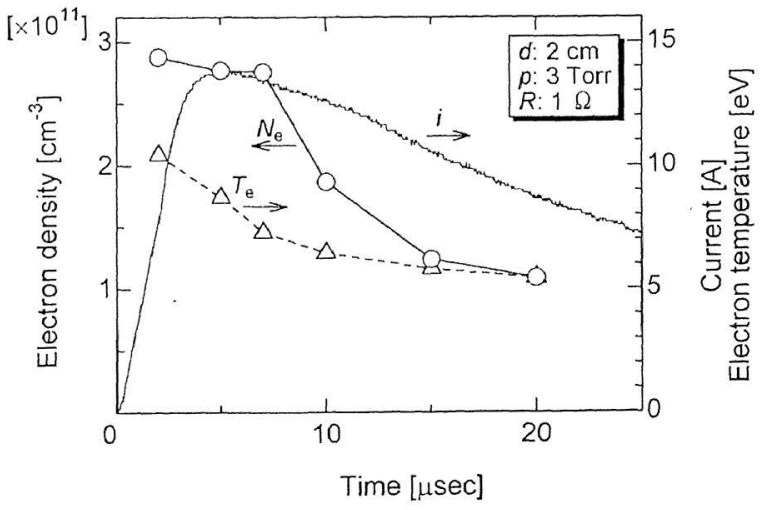

FIG. 4. Time dependence of electron density (solid) and electron temperature (dotted) of abnormal glow discharges. $P=3$ Torr, $d=2 \mathrm{~cm}$, and $R$ $=1 \Omega$. 
mode can be determined by division of the total glow current by the cross section of the positive column; it was calculated to be $89.9 \mathrm{~cm}^{2}$. Therefore, the electron densities of the positive column can be estimated to be $3.3 \times 10^{10} / \mathrm{cm}^{3}$ for normal glow mode $(I<3.7 \mathrm{~A})$ and $4.9 \times 10^{11} / \mathrm{cm}^{3}$ for abnormal glow mode $(I=144 \mathrm{~A})$.

Figure 4 shows the time variation of the electron density and the electron temperature measured with Langmuir probe at the middle between the electrodes. In this experiment, the gas pressure and the electrodes separation were kept at 3 Torr and $2 \mathrm{~cm}$, respectively. In this condition, the glow-toarc transition did not occur. The current, when the normal glow plasma covered the whole cathode, was 0.33 A from calculation of Eq. (2). The peak current of the pulse glow discharge is almost $14 \mathrm{~A}$, which is 40 times larger than the normal glow current $0.33 \mathrm{~A}$. Therefore, the discharge belongs to the abnormal glow. The electron density rapidly increases to almost $3 \times 10^{11} / \mathrm{cm}^{3}$ after a formation of the glow discharge. And then, the electron density becomes the value on the plateau of the time variation during $5 \mu \mathrm{s}$. The electron temperatures are in range from 5 to $10 \mathrm{eV}$.

The authors would like to thank Professor N. Sato of Iwate University, Professor H. Akiyama of Kumamoto Uni- versity, and Professor K. Yukimura of Doshisya University for their valuable discussions. The authors would like to thank Mr. S. Kato of Iwate University and Mr. Y. Nishimura of PEKURIS Co. for their cooperation.

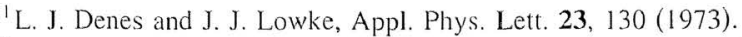

${ }^{2}$ S.-J. Park, C. J. Wagner, C. M. Herring, and J. G. Eden, Appl. Phys. Lett. 77, 199 (2000).

${ }^{3}$ J. Park, I. Henins, H. W. Hermann, G. S. Selwyn, J. Y. Jeong, R. F. Hicks, D. Shim, and C. S. Chang, Appl. Phy's. Lett. 76, 288 (2000).

${ }^{4}$ K. H. Schoenbach, A. El-Habachi, W. Shi, and M. Ciocca, Plasma Sources Sci. Technol. 6, 468 (1997).

${ }^{5}$ H. Barankova and L. Bardos, Appl. Phys. Lett. 76, 285 (2000).

${ }^{6}$ S. Takechi, S. Sugimoto, M. Kichi, K. Taaka, and S. Goto, in Proceedings of the Fifth International Workshop on Plasma-Based Ion Implantation, Kyoto, Japan, 13-16 December 1999, p. 17.

${ }^{7}$ K. Takaki, D. Kitamura, and T. Fujiwara, J. Phys. D 33, 1369 (2000).

${ }^{8}$ H. Akiyama, M. Kristiansen, H. Krompholz, and B. Mass, IEEE Trans. Plasma Sci. 16, 312 (1988).

${ }^{9}$ K. Takaki, A. Takahashi, and T. Fujiwara, J. Phys. D 33, 3060 (2000).

${ }^{10} \mathrm{Y}$. Asami, T. Dote, and Y. Suginuma, Houden Handbook (Corona Co., Tokyo, 1974), p. 110 (in Japanese).

${ }^{11}$ M. Cavenor and J. Meyer, Aust. J. Phys. 22, 155 (1969).

${ }^{12}$ S. Takeda, Kitai Houden no Kiso (Tokyo Denki, Tokyo, 1990), p. 35 (in Japanese). 